\title{
Essential Oils and Terpenes in Relation to Routes of Intake
}

\author{
Created by: Sachiko Koyama
}

Version received: 11 March 2020

The history of using essential oils is extremely long but the scientific studies at the chemical compound level are still in need. Essential oils have been used in three different ways: using diffusers, which will use the olfactory system primarily; drinking, which will use primarily the digestive system; and topical application, which will enter primarily through the skin. Various effects of essential oils have been reported so far. These diversity in the influences of essential oils could be due to 1) different chemical compounds or combination of different chemical compounds used, or 2) the differences in the routes (olfactory, skin, gut-intestine) the same essential oil activated, which suggest that different routes activate different receptors and thus different chemical signaling pathways. We need to consider these different routes as well as the differences in the chemical compounds in studying the influences of essential oils. It is also very important to conduct studies at the chemical compound level rather than using the whole essential oil in order to know which chemical compound affects. Using chemical compounds will enable precise control of the concentration in studying the effects as well.

\section{Introduction}

The history of using essential oils as the medical treatments or as the supplements to medical treatments goes far back to the ancient time in Egypt, which is even several thousand years ago. Depending on the countries and the cultures, essential oils have also been used in the religious ceremonies and for the relaxation for similarly long time period. This long history of using essential oils in the cultural events and the relaxation has most likely affected the images of essential oils in many people even still nowadays in a way that they are 'mysterious' or 'just for the relaxation', which presumably had disturbing influences to consider them as the subjects of scientific endeavor.

The beginning of $20^{\text {th }}$ century has become one of the turning points in the history of using essential oils. René-Maurice Gattefossé, a French chemist who worked at his family-owned perfume company, and not a clinician of holistic, alternative medicine, accidentally burnt his hand because of an explosion in the laboratory. He found that an essential oil of lavender suppressed the pain and the scar formation, which triggered him to start his researches on the essential oils from a chemist's viewpoint and also led him to coin the word 'aromatherapy' []․ The importance of his life-long studies on the essential oils is that he found the roles of terpenes in the effects of essential oils: "Essential oils contain constituents which possess almost the full range of chemical functions. The simplest are hydrocarbons, constituting the terpene family, of the type $p$-cymene, $\mathrm{C}_{10} \mathrm{H}_{14}$, which is similar to pinene, a constituent of oil of turpentine. The other constituents can almost all be classified as various stages in terpene development" ${ }^{[\underline{1}]}$. This can be called the starting point of the scientific studies on the effects of essential oils.

It has been already one century since then. There are increasing number of scientific studieson the effects of essential oils especially during the last few decades. Studies have found that essential oils have the effects on brain functions such as improving/decreasing cognitive function, depending on the types of essential oils [ㄹ][그, and some studies have found their effects on suppressing anxiety [4] . There are also studies showing their anti-nociceptive, anti-inflammatory, anti-convulsant, anti-oxidant, and 


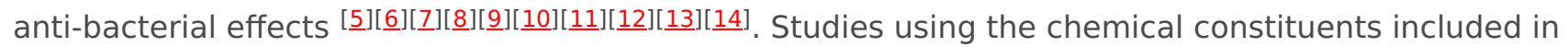
essential oils have shown that there are chemical compounds that have anti-cancer, anti-bacterial, antiviral, anti-inflammatory, anti-oxidant influences, and also these that enhance wound healing by

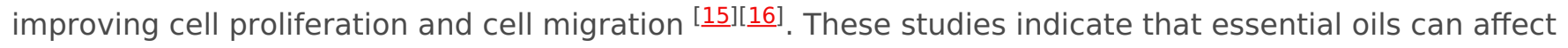
the central nervous system and also the peripheral system at cell levels. Such diversity in the influences suggests that essential oils may activate separate routes: these related to the signaling pathways related to the brain functions and these related to the signaling pathways related to the cell proliferation and cell migration, and apoptosis, and thus longevity of cells, in the peripheral systems.

The diversity in these effects makes us also consider whether the multiple types of methods in using essential oils have some roles. Essential oils are used 1) by inhaling, which will use mostly the olfactory system and to some extent the skin as the chemical compounds will reach the skin. 2) By topically applying essential oil on the skin, in which the major route will be through the skin and to a lesser extent through the olfactory system as the aroma will reach the olfactory system. 3) By drinking, in which the major route will be through the digestive system and, secondarily, because of the retro-nasal location of the nose, through the olfactory sensory system, as the aroma will reach the nose from the mouth and stomach. It is possible that, depending on the way of using essential oils, they activate different routes and, depending on the routes activated, they produce different effects. It is necessary to take into consideration how the essential oils were applied when the effects of essential oils were studied.

\section{Through the olfactory system}

Studies of essential oils using the method of inhalation are testing the influences through the olfactory system in main. Considering that the olfactory system has routes directly to the brain, this could be the pathway that affects brain functions.

Studies so far using essential oils have shown that there are differences in the impacts depending on the types of essential oils. For example, when human subjects were exposed to the aroma of lavender (Lavandula angustifolia) or ylang-ylang (Cananga odorata) through a diffuser, their working memory decreased and the reaction time was slower in the tasks that required memory and attention, whereas the aroma of rosemary (Rosmarinus officinalis), peppermint (Mentha piperita), common sage (Salvia

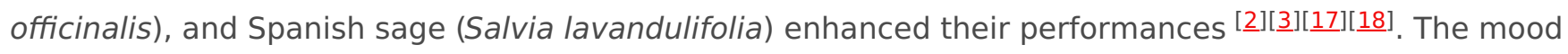
of the subjects were also more alert and more active and 'fresher' when they were exposed to the aroma of rosemary (Rosmarinus officinalis) than the lavender (Lavandula angustifolia) [2]][3][1] ] . These results suggest that it would be better to use lavender (Lavandula angustifolia) and ylang-ylang (Cananga odorata) for relaxation, and peppermint and rosemary in enhancing alertness and memory function.

Where are the aromas of the essential oils detected in the nasal cavity? Studies on the olfactory system of mice have found that there are multiple regions in the nasal cavity, which are involved in the olfactory sense: the main olfactory epithelium, the vomeronasal organ, the septal organ of Masera, and the Grüneberg ganglion [19][리]. In addition, there are separate sensory receptors expressed in other sensory neurons that reside in the main olfactory epithelium or the vomeronasal organ but carry different receptor types. These are the receptor guanylyl cyclase (GC-D) ${ }^{[21]}$ and trace amine-associated receptors (TAAR) $[\underline{22][23]}$ in the main olfactory epithelium and formyl peptide receptors in the vomeronasal organ. In case of humans, most likely, the main olfactory epithelium is the single functional olfactory system. The main olfactory epithelium is located at the upmost region in the nasal cavity. It is the area, which is closest to the brain, where the olfactory epithelium spreads on the nasal turbulence. In case of mice, there are about 1000 different types of olfactory receptors [24][르], whereas in case of humans, there are less than $400[\underline{25}][\underline{26}][\underline{27}][\underline{28}]$. Each olfactory neurons carry a single type of olfactory receptor, which enables the olfactory system to distinguish odors. The olfactory receptors are not homogeneously distributed in the olfactory epithelium, but instead, they are split in the areas constructing 4 zones' structure [29][이 . In addition, a new neural cell adhesion molecule, known as the olfactory cell adhesion molecule (OCAM), was found and the expression of OCAM showed zone 
differences, i.e., negative in Zone 1 but positive in Zones 2 to 4 in the main olfactory epithelium and in the main olfactory bulb (MOB), and positive in the apical region of the vomeronasal organ and rostral region of the accessory olfactory bulb $(\mathrm{AOB})$ but negative in the basal region of the vomeronasal organ and caudal $A O B$ [30]. Based on these findings, the names of Zone 1 to 4 of the olfactory epithelium are often called the Dorsal domain (Zone 1; OCAM negative) and the Ventral domain (Zones 2 to 4; OCAM positive). The axons from the olfactory neurons, which carry the same olfactory receptors, assemble at the MOB and generate a glomerulus. Recent studies have determined the types of odors and the locations of the glomeruli related to these odors in the olfactory bulb [31]. The glomeruli related to terpenes were found to be located in the ventral domain of the olfactory bulbs $[\underline{31]}$. This area is where the axons from the lateral/ventral areas reach, and this suggests that the area where terpenes in essential oils are sensed in the olfactory epithelium could be the lateral/ventral areas which project their axons from sensory neurons to the ventral domain of the MOB. This hypothesis is partially supported using three types of terpene compounds, cavone, 1,8-cineole, and limonene and the different responses generated that depend on the types of compounds. 1,8-Cineole and limonene generated responses in the lateral/ventral part of the olfactory epithelium whereas the responses to cavone included the dorsal region and the lateral/ventral region [32].

\section{Through the skin}

There are multiple types of receptors present in skin that can be involved in the pathways activated by the chemical constituents included in the essential oils: the olfactory receptors expressed in skin, the cannabinoid receptors (CB receptors), and the transient receptor potential channels (TRP channels).

The olfactory receptors expressed in the non-olfactory system including skin are not involved in the olfactory sense. There are some differences in their functions depending on the locations they are found, but so far they are found to be involved in the chemical reactions like chemotaxis [33], adjusting blood pressure ${ }^{[34]}$, and stimulating secretion of enzymes ${ }^{[34]}$. The tissues/organs or cells that these olfactory

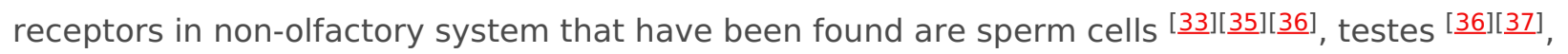

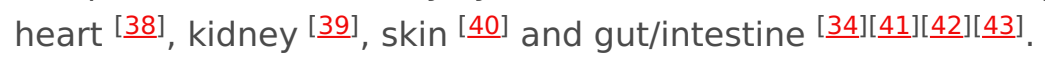

Recent studies have found that human olfactory receptor OR2AT4 is expressed in the epidermis of the skin, with the strongest expression in the basal layer, stratum basale, and that OR2AT4 becomes activated by sandalore, a synthetic sandalwood (Santalum) odorant [므] . Activation of OR2AT4 stimulated cell proliferation and migration, activation of CAMP-dependent pathways, and phosphorylation of extracellular signal-regulated kinases (Erk1/2) and p38 mitogen-activated protein kinases (p38 MAPK). OR2AT4 was later found to be expressed in the outer root sheath of hair follicles. Stimulation of OR2AT4 by sandalore suppressed apoptosis and extended hair growth and longevity [니][4], which suggests another possible use of OR2AT4, i.e., in avoiding thinning of hair.

Another human olfactory receptor, OR51E2, was found in human epidermal melanocytes [ㄴㄷ․ The ligand of OR51E2 was $\beta$-ionone, which is an aroma-odorant included in, for example, rose (Rosa) essential oils. Activation of OR51E2 by $\beta$-ionone stimulated melanin synthesis, suggesting the possibility of utilizing it in the "treatment of pigmentation disorders and proliferative pigment cell disorders such as melanoma" [46].

Human olfactory receptors OR2A4/7 and OR51B5 are also expressed in human skin and they are activated by the odorants cyclohexyl salicylate and isononyl alcohol, respectively [4]. Cyclohexyl salicylate has a rather strong flowery fragrance and is used in, for example, shampoos, shower gels, and soaps. Locations of expression of OR2A4/7 and OR51B5 were slightly different, although both were expressed in the epidermis: OR2A4/7 was expressed in keratinocytes in the supra-basal epidermis (stratum spinosum) and in the melanocytes in the basal layer (stratum basale), whereas OR51B5 was found expressed only in the keratinocytes of the supra-basal layer but not in the basal layer of the epidermis. Their impacts were also different. OR2A4/7 stimulated cytokinesis, cell migration, regeneration of keratinocytes, phosphorylation of AKT and Chk-2, and secretion of IL-1, whereas OR51B5 
stimulated cell migration, phosphorylation of Hsp27, AMPK1, and p38MAPK, and secretion of IL-6.

These studies have shown that, although there are some differences in the locations of expression and functions of these olfactory receptors, all of them similarly stimulate cell proliferation and cell migration when they become activated by their ligand aroma-odorants. In the morphology of skin, there are two areas, the basal epidermis and the upper bulge of hair follicles, where stem cells are produced to maintain skin homeostasis and hair regrowth, respectively. The results suggest the possibility that essential oils with the ligands for these olfactory receptors may become new clinical agents to enhance skin homeostasis and hair regrowth.

$\beta$-caryophyllene is a sesquiterpene included in various spice and herbs. For example, essential oils of oleoresin (Copaifera paupera) and lavender (Lavandula angustifolia) are well known to have betacaryophyllene in them. It has a smell, thus activates olfactory receptors, and recently it was found to be a ligand of cannabinoid receptor 2 (CB2) [48]. Recent studies have shown that topical application of $\beta$ caryophyllene improves re-epithelialization in the cutaneous wounds [ㄹ]. Essential oils of oleoresin (Copaifera paupera) which contains $\beta$-caryophyllene have shown the same effect $[\underline{50]}$. RNA sequencing and pathway analyses of skin exposed to $\beta$-caryophyllene showed that the pathways related to cell proliferation and cell migration were significantly activated (the sonic hedgehog pathway, the planar cell polarity signaling pathway, the fibroblast growth factor signaling pathway, and the Wnt $\beta$-catenin signaling pathway) [49]. Studies have found that hedgehog signaling is involved in epidermal homeostasis $[\underline{51}]$ and that sonic hedgehog secreted from sensory neurons surrounding the hair follicle bulge stimulates Gli1+ (a hair follicle bulge marker) cells in the upper hair follicle bulge to convert to multipotent stem cells which will migrate to the epidermis and contribute to re-epithelialization [52]. These studies suggest that $\beta$-caryophyllene application improves re-epithelialization through enhancing the conversion of hair follicle bulge stem cells into multipotent stem cells and stimulating their migration towards the basal epidermis, and, from there, towards the wound bed. This is the first study showing the effects of a chemical constituent of essential oils at the level of gene expressions and molecular analyses.

\section{The gut and intestine}

Much less have been studied focusing on the influences of essential oils through the gut and intestine. There are some recent studies, which have found that the chemical compounds in orange essential oils, i.e., limonene, linalool, and citral, enhance the microbiota, especially Lactobacillus, in the gut of mice, and limonene had the strongest impact $[\underline{53}$. The olfactory receptor for limonene, Olfr56 (human

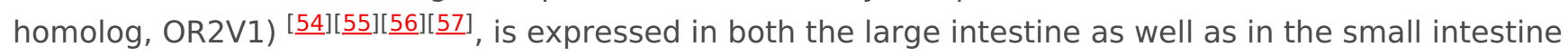
in mice. Olfactory receptor Olfr78 (human homolog, OR51E2) was also found expressed in some, but not all, of the enteroendocrine cells in the colon of mice, and propionate was found as their ligand [42]. In a separate study, Olfr78 responded to acetate and propionate ${ }^{[34]}$. Propionate is an important nutrient generated by bacteria, which stimulates secretion of satiety-inducing hormones that can adjust energy homeostasis [57]. These studies suggest that essential oils may be involved in regulating energy homeostasis by enhancing the microbiota, which will stimulate the secretion of propionate, which is the ligand of olfactory receptors. We still need more studies in detail to determine the influences of chemical constituents included in essential oils.

\section{Factors that affect the effects of essential oils and terpenes}

Despite the long history of using essential oils, there have been rather few scientific studies on them. Needless to say, there have been few studies taking consideration on the routes (olfactory, skin, gutintestine), receptors, gene expressions, and signaling pathways that they generate the effects. What is important in the studies on odorous chemicals is the specific cautions required in handling them so that the conditions are precisely controlled. Odorous chemical compounds has certain volatility, and, depending on their volatility, the concentration can easily change in the samples to test. Depending on the type of material used in preparing/diluting the samples, the chemical compounds can attach to the surface of the container easily, which will make the concentration decreased in the samples to be 
tested. It is necessary to know the purity of the samples and what else are included, if the purity is less than $99.9 \%$, because the small portion of the other chemical compounds may affect the results.

When the whole essential oil, and not the single chemical compound, is used in the studies, the possibilities of differences in the combination of chemical compounds included and the differences in their percentage can be much larger than using single chemical compound. Although there are some essential oils with rather small numbers of chemical compound constituents (for example, about 25 in the case of guaicawood (Bulnesia sarmienti) oil), most essential oils have 100 to 250 different chemical compound constituents, and some contain 300 to $400{ }^{[58]}$. In the very popular lavender (Lavandula angustifolia) and rosemary (Rosmarinus officinalis) oils, studies have identified 505 and 450 chemical compounds, respectively. Comparison of the major constituents of the same essential oil in several studies shows that the chemical compounds listed as major constituents vary. The amount of these major constituents varies depending on the part of the plant used, e.g., old leaves versus young leaves, different seasons, geographical locations, and even the weather depending on the year. This makes it critical to measure the constituents of the essential oils used in experiments, to control the exposure methods (inhalation vs. capsule digestion vs. topical application) as well as to state the environmental conditions which can affect the exposure (temperature, humidity, air flow). The data also indicate the importance of testing the effects of pure single chemical compounds contained in essential oils. This will make experiments comparable and help to determine the influences of each chemical compound and the concentration dependency of its effect.

\section{References}

1. Gattefossé, R.-M.. Gattefossé's Aromatherapy; Tisserand, R.B. (translated), Eds.; The C. W. Daniell Co., Ltd. (translated version): London (translated version), 1993 (original 1937); pp. 176.

2. Mark Moss; Jenny Cook; K. Wesnes; Paul Duckett; AROMAS OF ROSEMARY AND LAVENDER ESSENTIAL OILS DIFFERENTIALLY AFFECT COGNITION AND MOOD IN HEALTHY ADULTS. International Journal of Neuroscience 2002, 113, 15-38, 10.1080/00207450390161903.

3. Mark Moss; Steven Hewitt; Lucy Moss; K. Wesnes; MODULATION OF COGNITIVE PERFORMANCE AND MOOD BY AROMAS OF PEPPERMINT AND YLANG-YLANG. International Journal of Neuroscience 2007, 118, 59-77, 10.1080/00207450601042094.

4. Hiroki Harada; Hideki Kashiwadani; Yuichi Kanmura; Tomoyuki Kuwaki; Linalool Odor-Induced Anxiolytic Effects in Mice. Frontiers in Behavioral Neuroscience 2018, 12, 241, 10.3389/fnbeh.2018.00241.

5. Jackson Roberto Guedes Da Silva Almeida; Camila De Souza Araújo; Ana Paula De Oliveira; Rafaely Nascimento Lima; Péricles Barreto Alves; Tâmara Coimbra Diniz; Chemical constituents and antioxidant activity of the essential oil from leaves of Annona vepretorum Mart. (Annonaceae). Pharmacognosy Magazine 2014, 11, 615-618, 10.4103/0973-1296.160462.

6. Tâmara Coimbra Diniz; R. G. Oliveira-Júnior; Maria Alice Miranda Bezerra Medeiros; Mariana Gama E Silva; Roxana Braga De Andrade Teles; Paula Dos Passos Menezes; Bruna Maria Hipólito De Sousa; Luiza Abrahão Frank; Adriano Antunes De Souza Araújo; Mairim R. Serafini; et al.Sílvia Stanisçuaski GuterresCarlos Eduardo Pereira NunesMarcos SalvadorJackson Roberto Guedes Da Silva Almeida Anticonvulsant, sedative, anxiolytic and antidepressant activities of the essential oil of Annona vepretorum in mice: Involvement of GABAergic and serotonergic systems.. Biomedicine \& Pharmacotherapy 2019,111, 1074-1087, 10.1016/j.biopha.2018.12.114.

7. Mohamad Sanad Abu-Darwish; Célia Cabral; M.J. Gonçalves; Carlos Cavaleiro; Maria Teresa Cruz; Thomas Efferth; L. Salgueiro; Artemisia herba-alba essential oil from Buseirah (South Jordan): Chemical characterization and assessment of safe antifungal and anti-inflammatory doses. Journal of Ethnopharmacology 2015, 174, 153-160, 10.1016/j.jep.2015.08.005.

8. Soumaya Bourgou; Iness Bettaieb Rebey; Khaoula Mkadmini; Hiroko Isoda; Riadh Ksouri; Wided Megdiche-Ksouri; Iness Bettaieb; LC-ESI-TOF-MS and GC-MS profiling of Artemisia herba-alba and evaluation of its bioactive properties. Food Research International 2017, 99, 702-712, 10.1016/j.foodres.2017.06.009.

9. Tarik Mohamed; Abeer A. Abd El Aty; Abdelaaty A. Shahat; Nahla S. Abdel-Azim; Khaled Shams; Abdelsamed I. 
ElShamy; Mehawed M. Ahmed; Sabry H. H. Youns; Taher M. El-Wassimy; Sayed A. El-Toumy; et al.Mohamed Elamir Hegazy New antimicrobial metabolites from the medicinal herb Artemisia herba-Alba.. Natural Product Research 2019, 31, 1-9, 10.1080/14786419.2019.1647430.

10. Gerardo D. Anaya-Eugenio; Isabel Rivero-Cruz; Robert Bye; Edelmira Linares; Rachel Mata; Antinociceptive activity of the essential oil from Artemisia ludoviciana. Journal of Ethnopharmacology 2016, 179, 403-411, 10.1016/j.jep.2016.01.008.

11. Khaled Elmassry; Ahmed El-Ghorab; Amr Farouk; Antioxidant activity and volatile components of Egyptian Artemisia judaica L.. Food Chemistry 2002, 79, 331-336, 10.1016/s0308-8146(02)00164-4.

12. Mohamad Sanad Abu-Darwish; Célia Cabral; M.J. Gonçalves; Carlos Cavaleiro; M.T. Cruz; Ali Zulfiqar; I.A. Khan; Thomas Efferth; Lígia Salgueiro; Chemical composition and biological activities of Artemisia judaica essential oil from southern desert of Jordan. Journal of Ethnopharmacology 2016, 191, 161-168, 10.1016/j.jep.2016.06.023.

13. Hyun-Jung Park; Eun-Ju Lim; Rong Jie Zhao; Sa Rang Oh; Ji Wook Jung; Eun-Mi Ahn; Eun Sook Lee; Jin Suk Koo; Hee Young Kim; Suchan Chang; et al.Hyun Soo ShimKwang Joong KimYoung S. GwakChae Ha Yang Effect of the fragrance inhalation of essential oil from Asarum heterotropoides on depression-like behaviors in mice.. BMC Complementary and Alternative Medicine 2015, 15, 43, 10.1186/s12906-015-0571-1.

14. A.S.M. Tanbirul Haque; Jin Nam Moon; P.S. Saravana; Adane Tilahun; Byung-Soo Chun; Composition of Asarum heterotropoides var. mandshuricum radix oil from different extraction methods and activities against human body odor-producing bacteria. Journal of Food and Drug Analysis 2016, 24, 813-821, 10.1016/j.jfda.2016.04.006.

15. Jovana Petrović; Dejan Stojković; Marina Soković; Terpene core in selected aromatic and edible plants: Natural health improving agents.. Advances in Food and Nutrition Research 2019, 90, 423-451, 10.1016/bs.afnr.2019.02.009.

16. Aline Cristina Guimarães; Leandra Meireles; Mayara Fumiere Lemos; Marco Cesar Cunegundes Guimarães; Denise C. Endringer; Marcio Fronza; R. Scherer; Antibacterial Activity of Terpenes and Terpenoids Present in Essential Oils.. Molecules 2019, 24, 2471, 10.3390/molecules24132471.

17. Winai Sayorwan; Nijsiri Ruangrungsi; Teerut Piriyapunyporn; Tapanee Hongratanaworakit; Naiphinich Kotchabhakdi; Vorasith Siripornpanich; Effects of Inhaled Rosemary Oil on Subjective Feelings and Activities of the Nervous System. Scientia Pharmaceutica 2012, 81, 531-542, 10.3797/scipharm.1209-05.

18. Marco Miroddi; Michele Navarra; Maria C. Quattropani; Fabrizio Calapai; Sebastiano Gangemi; Gioacchino Calapai; Systematic Review of Clinical Trials Assessing Pharmacological Properties of Salvia Species on Memory, Cognitive Impairment and Alzheimer's Disease. CNS Neuroscience \& Therapeutics 2014, 20, 485-495, 10.1111/cns.12270.

19. Heinz Breer; Joerg Fleischer; J. Strotmann; The sense of smell: multiple olfactory subsystems. .Cellular and Molecular Life Sciences 2006, 63, 1465-1475, 10.1007/s00018-006-6108-5.

20. Ma, M.. Multiple olfactory subsystems convey various sensory signals. In "The Neurobiology of Olfaction." ; Menini, A., Eds.; CRC Press/Taylor \& Francis: Boca Raton , 2010; pp. Chapter 9.

21. H. J. Fülle; R. Vassar; D. C. Foster; Ruey-Bing Yang; R. Axel; D. L. Garbers; A receptor guanylyl cyclase expressed specifically in olfactory sensory neurons.. Proceedings of the National Academy of Sciences1995, 92, 3571-3575, 10.1073/pnas.92.8.3571.

22. Stephen D. Liberles; Linda B. Buck; A second class of chemosensory receptors in the olfactory epithelium.Nature 2006, 442, 645-650, 10.1038/nature05066.

23. Stephen D. Liberles; Trace Amine-associated Receptors Are Olfactory Receptors in Vertebrates.Annals of the New York Academy of Sciences 2009, 1170, 168-172, 10.1111/j.1749-6632.2009.04014.x.

24. Linda Buck; Richard Axel; A novel multigene family may encode odorant receptors: A molecular basis for odor recognition. Cell 1991, 65, 175-187, 10.1016/0092-8674(91)90418-x.

25. Peter Mombaerts; MOLECULAR BIOLOGY OF ODORANT RECEPTORS IN VERTEBRATES. Annual Review of Neuroscience 1999, 22, 487-509, 10.1146/annurev.neuro.22.1.487.

26. Peter Mombaerts; Seven-Transmembrane Proteins as Odorant and Chemosensory Receptors.Science 1999, 286, 707-711, 10.1126/science.286.5440.707.

27. Stuart Firestein; How the olfactory system makes sense of scents. Nature 2001, 413, 211-218, 10.1038/35093026.

28. Barry W. Ache; Janet M. Young; Olfaction: Diverse Species, Conserved Principles.Neuron 2005, 48, 417-430, 10.1016/j.neuron.2005.10.022.

29. Kensaku Mori; H Von Campenhause; Yoshihiro Yoshihara; Zonal organization of the mammalian main and accessory olfactory systems.. Philosophical Transactions of the Royal Society B: Biological Sciences2000, 355, 1801-1812, 10.1098/rstb.2000.0736.

30. Yoshihiro Yoshihara; Miwa Kawasaki; Atsushi Tamada; Hiroko Fujita; Hideyuki Hayashi; Hiroyuki Kagamiyama; Kensaku Mori; OCAM: A New Member of the Neural Cell Adhesion Molecule Family Related to Zone-to-Zone Projection of Olfactory and Vomeronasal Axons. The Journal of Neuroscience 1997, 17, 5830-5842, 10.1523/JNEUROSCI.17-15-05830.1997.

31. Kensaku Mori; Hitoshi Sakano; How Is the Olfactory Map Formed and Interpreted in the Mammalian Brain? Annual Review of Neuroscience 2011, 34, 467-499, 10.1146/annurev-neuro-112210-112917.

32. J W Scott; T Brierley; A functional map in rat olfactory epithelium..Chemical Senses 1999, 24, 679-690, 
10.1093/chemse/24.6.679.

33. Marc Spehr; Günter Gisselmann; Alexandra Poplawski; Jeffrey A. Riffell; Christian H. Wetzel; Richard K. Zimmer; Hanns Hatt; Identification of a Testicular Odorant Receptor Mediating Human Sperm Chemotaxis. Science 2003, 299, 2054-2058, 10.1126/science.1080376.

34. Jennifer L. Pluznick; Ryan J. Protzko; Haykanush Gevorgyan; Zita Peterlin; Arnold Sipos; Jinah Han; Isabelle Brunet; La-Xiang Wan; Federico Rey; Tong Wang; et al.Stuart J. FiresteinMasashi Yanagisawajeffrey I. GordonAnne EichmannJanos Peti-PeterdiMichael J. Caplan Olfactory receptor responding to gut microbiota-derived signals plays a role in renin secretion and blood pressure regulation. Proceedings of the National Academy of Sciences2013, 110, 4410-4415, 10.1073/pnas.1215927110.

35. M. Parmentier; Frédéric Libert; Stephane Schurmans; Serge Schiffmann; Anne Lefort; Dominique Eggerickx; Catherine Ledent; Catherine Mollereau; Catherine Gérard; Jason Perret; et al.Anton GrootegoedGilbert VassartCatherine $\mathrm{G}$ Expression of members of the putative olfactory receptor gene family in mammalian germ cells. Nature 1991, 355, 453-455, 10.1038/355453a0.

36. Nanaho Fukuda; Kentaro Yomogida; Masaru Okabe; Kazushige Touhara; Functional characterization of a mouse testicular olfactory receptor and its role in chemosensing and in regulation of sperm motility. Journal of Cell Science 2004, 117, 5835-5845, 10.1242/jcs.01507.

37. Nanaho Fukuda; Kazushige Touhara; Developmental expression patterns of testicular olfactory receptor genes during mouse spermatogenesis. Genes to Cells 2005, 11, 71-81, 10.1111/j.1365-2443.2005.00915.x.

38. Sung-Hee Kim; Yeo Cho Yoon; Ae Sin Lee; Nana Kang; Jaehyung Koo; Mee-Ra Rhyu; Jae Ho Park; Expression of human olfactory receptor $10 \mathrm{~J} 5$ in heart aorta, coronary artery, and endothelial cells and its functional role in angiogenesis. Biochemical and Biophysical Research Communications 2015, 460, 404-408, 10.1016/j.bbrc.2015.03.046.

39. Jennifer L. Pluznick; Ng-Jing Zou; Xiaohong Zhang; Qingshang Yan; Diego J. Rodriguez-Gil; Christoph Eisner; Erika Wells; Charles A. Greer; Tong Wang; Stuart Firestein; et al.Jurgen SchnermannMichael J. Caplan Functional expression of the olfactory signaling system in the kidney. Proceedings of the National Academy of Sciences2009, 106, 2059-2064, 10.1073/pnas.0812859106.

40. Daniela Busse; Philipp Kudella; Nana-Maria Grüning; Günter Gisselmann; S. Ständer; Thomas Luger; Frank Jacobsen; Lars Steinsträßer; Ralf Paus; Paraskevi Gkogkolou; et al.Markus BöhmHanns HattHeike Benecke A Synthetic Sandalwood Odorant Induces Wound-Healing Processes in Human Keratinocytes via the Olfactory Receptor OR2AT4. Journal of Investigative Dermatology 2014, 134, 2823-2832, 10.1038/jid.2014.273.

41. Thomas Braun; Petra Voland; Lars Kunz; Christian Prinz; Manfred Gratzl; Enterochromaffin Cells of the Human Gut: Sensors for Spices and Odorants. Gastroenterology 2007, 132, 1890-1901, 10.1053/j.gastro.2007.02.036.

42. Joerg Fleischer; Rosolino Bumbalo; Verena Bautze; Joerg Strotmann; Heinz Breer; Expression of odorant receptor Olfr78 in enteroendocrine cells of the colon. Cell and Tissue Research 2015, 361, 697-710, 10.1007/s00441-0152165-0.

43. Ki-Suk Kim; In-Seung Lee; Kang-Hoon Kim; Jiyoung Park; Yumi Kim; Jeong-Hee Choi; Jin-Sung Choi; Hyeung-Jin Jang; Activation of intestinal olfactory receptor stimulates glucagon-like peptide-1 secretion in enteroendocrine cells and attenuates hyperglycemia in type 2 diabetic mice. Scientific Reports 2017, 7, 13978, 10.1038/s41598-017-14086-5.

44. Jérémy Chéret; Marta Bertolini; Leslie Ponce; Janin Lehmann; Teresa Tsai; Majid Alam; Hanns Hatt; Ralf Paus; Olfactory receptor OR2AT4 regulates human hair growth. Nature Communications 2018, 9, 3624, 10.1038/s41467018-05973-0.

45. Lian Gelis; Nikolina Jovancevic; Sophie Veitinger; Bhubaneswar Mandal; Hans-Dieter Arndt; Eva Neuhaus; Hanns Hatt; Functional Characterization of the Odorant Receptor $51 E 2$ in Human Melanocytes*. Journal of Biological Chemistry 2016, 291, 17772-17786, 10.1074/jbc.M116.734517.

46. Barbara Pavan; Alessandro Dalpiaz; Odorants could elicit repair processes in melanized neuronal and skin cells. Neural Regeneration Research 2016, 12, 1401-1404, 10.4103/1673-5374.215246.

47. Teresa Tsai; Sophie Veitinger; Irina Peek; Daniela Busse; Josephine Eckardt; Dilyana Vladimirova; Nikolina Jovancevic; Sebastian Wojcik; Günter Gisselmann; Janine Altmüller; et al.S. StänderThomas LugerRalf PausJeremy CheretHanns Hatt Two olfactory receptors-OR2A4/7 and OR51B5-differentially affect epidermal proliferation and differentiation. Experimental Dermatology 2016, 26, 58-65, 10.1111/exd.13132.

48. Jürg Gertsch; Marco Leonti; Stefan Raduner; Ildikó Rácz; Jian-Zhong Chen; Xiang-Qun Xie; Karl-Heinz Altmann; Meliha Karsak; Andreas Zimmer; Beta-caryophyllene is a dietary cannabinoid. Proceedings of the National Academy of Sciences 2008, 105, 9099-9104, 10.1073/pnas.0803601105.

49. Sachiko Koyama; Anna Purk; Manpreet Kaur; Helena A. Soini; Milos V. Novotny; Keith Davis; C. Cheng Kao; Hiroaki Matsunami; Anthony Mescher; Beta-caryophyllene enhances wound healing through multiple routes.. PLOS ONE 2019, 14, e0216104, 10.1371/journal.pone.0216104.

50. Jorge Luis Amorim; Janaína De Barros Figueiredo; Ana Cláudia Fernandes Amaral; Eliane Gouvêa De Oliveira Barros; Célia Palmero; Maria Athana Mpalantinos; Aline De Souza Ramos; José Luiz Pinto Ferreira; Jefferson Silva; Claudia Benjamim; et al.Silvia Luciane BassoLuiz Eurico NasciuttiPatricia Dias Fernandes Wound healing properties of 
Copaifera paupera in diabetic mice. PLOS ONE 2017, 12, e0187380, 10.1371/journal.pone.0187380.

51. Yoshinori Abe; Nobuyuki Tanaka; Roles of the Hedgehog Signaling Pathway in Epidermal and Hair Follicle Development, Homeostasis, and Cancer. Journal of Developmental Biology 2017, 5, 12, 10.3390/jdb5040012.

52. Isaac Brownell; Elizabeth Guevara; C. Brian Bai; Cynthia A. Loomis; Alexandra L. Joyner; Nerve-Derived Sonic Hedgehog Defines a Niche for Hair Follicle Stem Cells Capable of Becoming Epidermal Stem Cells. Cell Stem Cell 2011, 8, 552-65, 10.1016/j.stem.2011.02.021.

53. Lin Wang; Yan Zhang; Gang Fan; Jing-Nan Ren; Lu-Lu Zhang; Si-Yi Pan; Effects of orange essential oil on intestinal microflora in mice. Journal of the Science of Food and Agriculture2019, 99, 4019-4028, 10.1002/jsfa.9629.

54. Dietmar Krautwurst; King-Wai Yau; Randall R Reed; Identification of ligands for olfactory receptors by functional expression of a receptor library.. Cell 1998, 95, 917-926, 10.1016/s0092-8674(00)81716-x.

55. Xinmin Zhang; Ivan Rodriguez; Peter Mombaerts; Stuart Firestein; Odorant and vomeronasal receptor genes in two mouse genome assemblies. Genomics 2004, 83, 802-811, 10.1016/j.ygeno.2003.10.009.

56. Paul A. Godfrey; B. Malnic; Linda B. Buck; The mouse olfactory receptor gene family.Proceedings of the National Academy of Sciences 2004, 101, 2156-2161, 10.1073/pnas.0308051100.

57. Racha El Hage; Emma Hernandez-Sanabria; Marta Calatayud Arroyo; Ruben Props; Tom Van De Wiele; PropionateProducing Consortium Restores Antibiotic-Induced Dysbiosis in a Dynamic in vitro Model of the Human Intestinal Microbial Ecosystem.. Frontiers in Cellular and Infection Microbiology 2019, 10, 1206, 10.3389/fmicb.2019.01206.

58. Anton C. De Groot; Erich Schmidt; Essential Oils, Part III.Dermatitis 2015, 27, 161-169, 10.1097/der.0000000000000193.

\section{Keywords}

essential oils; routes; olfactory system; skin; gut-intestine

(c) 2020 by the author(s). Distribute under a Creative Commans CC BY license 